\title{
Tenue dans le temps des composants des pompes-turbines soumises à de nombreux changements de régimes journaliers
}

\section{Life of pumps-turbine components \\ undergoing numerous changes in operating conditions during daily service}

\author{
André Lacoste \\ Division Matériel \\ au Service de la Production Hydraulique d'EDF, Paris
}

Parc EDF de groupes de pompage-turbinage

L'expérience d'Electricité de France concernant les centrales de Transfert d'Energie de grande puissance est relativement récente puisque la première pompe-turbine de plus de 50 MW a démarré à Vouglans en 1973.

Le tableau 1 donne les principales caractéristiques des 5 centrales de pompage-turbinage exploitées actuellement par EDF et comportant des groupes de plus de
$50 \mathrm{MW}$ qui sont tous des groupes réversibles. La Centrale du Cheylas est actuellement dans sa phase de démarrage.

D'autres suivront avec Montezic, Grand Maison, Super-Bissorte, Redenat.

Les caractéristiques de Vouglans, en particulier la capacité de son réservoir et sa dépendance du réseau $60 \mathrm{kV}$, en font un groupe de transfert saisonnier, d'ailleurs peu utilisé actuellement en pompage.

Notre expérience en ce qui conceme les problèmes posés par les pompes-turbines très sollicitées se limite

Tableau 1 - Stations de transfert d'énergie comportant des machines de puissance unitaire supérieure à $50 \mathrm{MW}$ exploitées par Electricité de France ou en projet

\begin{tabular}{|c|c|c|c|c|c|c|c|c|c|c|c|c|c|c|c|}
\hline \multirow{2}{*}{ Usines } & \multirow{2}{*}{$\begin{array}{l}\text { Annice } \\
\text { de } \\
\text { mise en } \\
\text { scruice }\end{array}$} & \multirow{2}{*}{$\begin{array}{l}\text { ruissance } \\
\text { nominale } \\
\text { totale } \\
\text { en } \\
\text { turbine } \\
\text { co Ma: }\end{array}$} & \multirow{2}{*}{$\begin{array}{c}\text { lombre de } \\
\text { groupes } \\
\text { et } \\
\text { type }\end{array}$} & \multicolumn{3}{|c|}{ Fonctionnement en furbinc } & \multicolumn{3}{|c|}{ ronctionnement en Pompe } & \multirow{2}{*}{ 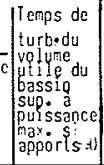 } & \multirow{2}{*}{$\begin{array}{l}\text { Vitesse } \\
\text { de } \\
\text { rotation } \\
\text { en } \mid \mathrm{r} / \mathrm{min}\end{array}$} & \multirow{2}{*}{$\begin{array}{l}\text { Cingr rayge } \\
\text { en } \\
\text { Pompe }\end{array}$} & \multirow{2}{*}{$\begin{array}{l}\text { Worche } \\
\text { en } \\
\text { Compenm } \\
\text { sateur } \\
\text { synchrone }\end{array}$} & \multicolumn{2}{|c|}{$\begin{array}{l}\text { Temps do dimarraye à parlir } \\
\text { de l'arré jusqu'au couplage }\end{array}$} \\
\hline & & & & $\begin{array}{l}\text { II de } \\
\text { chute } \\
\text { nelte n. } \\
\text { extrênes }\end{array}$ & $\begin{array}{l}0 \\
\text { unitaire } \\
\mathrm{m} 3 / \mathrm{s}\end{array}$ & $\begin{array}{l}\text { uissance } \\
\text { uni taire } \\
\text { fournie }\end{array}$ & $\begin{array}{l}\text { il d'elém } \\
\text { valion } \\
\text { totale } \\
\text { en m. }\end{array}$ & $\begin{array}{c}0 \\
\text { unitaire } \\
\text { m3/s }\end{array}$ & $\mid \begin{array}{l}\text { Puissance } \\
\text { unitaire } \\
\text { absorbée }\end{array}$ & & & & & $\begin{array}{l}\text { En Yurbine } \\
\text { juscu'au } \\
\text { couplange }\end{array}$ & $\begin{array}{l}\text { En Ponpe } \\
\text { Jusqu'à l'ou- } \\
\text { verture du } \\
\text { vannaiye }\end{array}$ \\
\hline VUIGLAIS & 16.4 .73 & 60 & liv & $\begin{array}{r}100,2 \\
73,2\end{array}$ & $\begin{array}{l}69 \\
54,9\end{array}$ & $\begin{array}{l}62 \\
34,3\end{array}$ & $\begin{array}{l}29,33 \\
80,4,0\end{array}$ & $\begin{array}{l}48 \\
68,6\end{array}$ & $\begin{array}{l}52,6 \\
52,3\end{array}$ & $1845 \mathrm{~h}$ & 150 & \begin{tabular}{l|} 
a) dos-dodos \\
avec Iurb. \\
voisine roue \\
noyé \\
b) asynchron \\
rove noyec
\end{tabular} & Non & $3 \mathrm{mn}$ & $\begin{array}{l}\text { a) } 7 \mathrm{mn} \\
\text { b) } 1 \mathrm{mn} ; \mathrm{s}\end{array}$ \\
\hline Sle-CROIK & 1975 & 53 & Irv & $\begin{array}{l}76,87 \\
60,50\end{array}$ & $\begin{array}{l}87,60 \\
75,10\end{array}$ & $\begin{array}{l}58,66 \\
39,31\end{array}$ & $\begin{array}{l}78,78 \\
63,88\end{array}$ & $\begin{array}{l}54,2 \\
70,6\end{array}$ & $\begin{array}{l}46,2 \\
48,80\end{array}$ & $980 \mathrm{~h}$ & 166,7 & $\begin{array}{l}\text { pos-à-dos } \\
\text { pvec Turbine } \\
\text { voisine } \\
\text { roue noyée }\end{array}$ & Non & $5 \pi n$ & $6 \mathrm{mn} 20 \mathrm{~s}$ \\
\hline ryill & 1976 & 720 & $45 \mathrm{y}$ & $\begin{array}{l}246,5 \\
219\end{array}$ & $\begin{array}{r}102 \\
89\end{array}$ & $\begin{array}{l}198 \\
164\end{array}$ & $\begin{array}{l}243 \\
222\end{array}$ & $\begin{array}{l}64 \\
74\end{array}$ & $\begin{array}{l}175 \\
184_{1}\end{array}$ & $5 \ln 20$ & 300 & $\begin{array}{l}\text { l-2-3 } \\
\text { hoteur lancen } \\
\text { fit asynchr. } \\
\text { folor piscine } \\
\text { roue dénoyée }\end{array}$ & Oui & $\left\{\begin{array}{l}2 \operatorname{man} 10 \mathrm{~s} \\
2 \operatorname{mon} 10 \mathrm{~s}\end{array}\right.$ & $\begin{cases}61-2-3 & 12 \mathrm{~m} \\
64 & \end{cases}$ \\
\hline la cocue & $1977^{*}$ & 301,6 & $\begin{array}{c}\text { if } r u \\
\text { mult lét ages } \\
\text { sans vannage }\end{array}$ & $\begin{array}{l}125 \\
894\end{array}$ & $\begin{array}{r}10,3 \\
8,5\end{array}$ & $\begin{array}{l}79,8 \\
63,1\end{array}$ & $\begin{array}{l}948 \\
917\end{array}$ & $\begin{array}{l}8 \\
8,5\end{array}$ & $\begin{array}{l}75 \\
80,8\end{array}$ & $14 \mathrm{~h}$ & 600 & $\begin{array}{l}\text { tsynchrone } \\
\text { roue noyće }\end{array}$ & Non & $1 \mathrm{mn} 55$ & $\operatorname{lnn} 45 \mathrm{~s}$ \\
\hline $\begin{array}{l}(\cdot) \text { Premier } \\
\text { Quatriter } \\
\text { Exploila }\end{array}$ & $\begin{array}{c}\text { roupe en } \\
\text { Groupe en } \\
\text { ion norma }\end{array}$ & $\left\{\begin{array}{l}75 \\
i^{\prime}: 77 \\
\text { depuis }\end{array}\right.$ & delobre 1978. & $\begin{array}{l}256,2 \\
232,8\end{array}$ & $\begin{array}{l}11^{4} \\
107,4\end{array}$ & $\begin{array}{l}252 \\
214\end{array}$ & $\begin{array}{l}267,55 \\
21,7,80\end{array}$ & $\begin{array}{l}79,5 \\
89\end{array}$ & $\begin{array}{l}229,3 \\
236,7\end{array}$ & $5 h$ & 300 & $\begin{array}{l}\text { Asynchrone } \\
\text { B tension } \\
\text { éduite rove } \\
\text { énoyée }\end{array}$ & oui & $2 \mathrm{mn} 10 \mathrm{~s}$ & $3 \mathrm{mn} 20 \mathrm{~s}$ \\
\hline
\end{tabular}

LA HOUILLE BLANCHE/N $1 / 2-1980$ 
donc aux trois centrales de Ste-Croix, Revin et La Coche dont les 9 machines ont tout de même fonctionné en temps cumulés environ pendant :

$14630 \mathrm{~h}$ en Turbine

$19623 \mathrm{~h}$ en Pompe

$1065 \mathrm{~h}$ en Compensateur

(Revin seulement)

Soit au Total $35318 \mathrm{~h}$

En fait, nous allons voir que ces temps cumulés de fonctionnement ne sont pas très représentatifs des problèmes posés en exploitation par ces machines.

\section{Mode d'exploitation actuel}

Quel est le mode d'exploitation actuel des centrales de Transfert d'Energie?

La figure 2 représente le diagramme de fonctionnement de la Centrale de Revin pour la journée du 20 décembre 1978. On peut voir qu'il y a eu ce jour-là 22 changements d'état, démarrage, arrêt, passage de turbine en compensateur, de compensateur en pompe...

D'autre part, on peut voir que les puissances actives et réactives délivrées par les groupes sont très variables tout le long de la journée.

Même constatation sur le diagramme de la figure 3 concernant $\mathrm{La}$ Coche dont les groupes, rappelons-le, ne

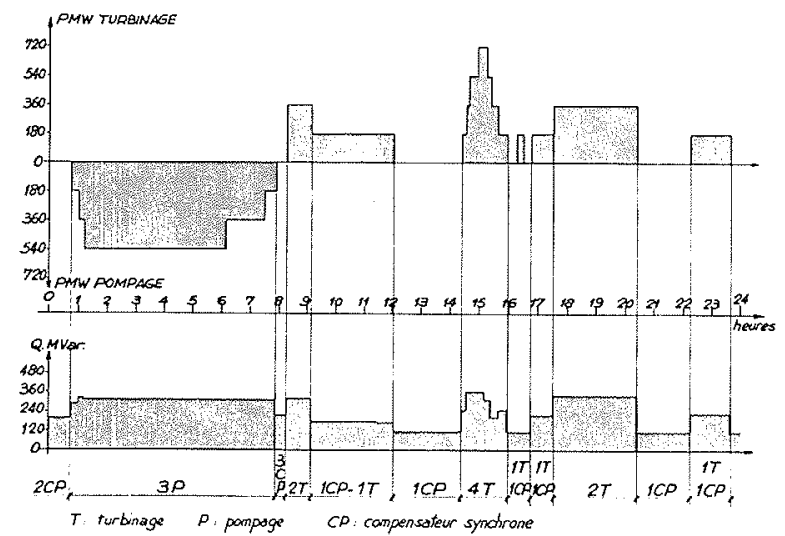

Figure 2 - Fonctionnement des groupes de Revin pendant la journée du Mercredi 20 décembre 1978

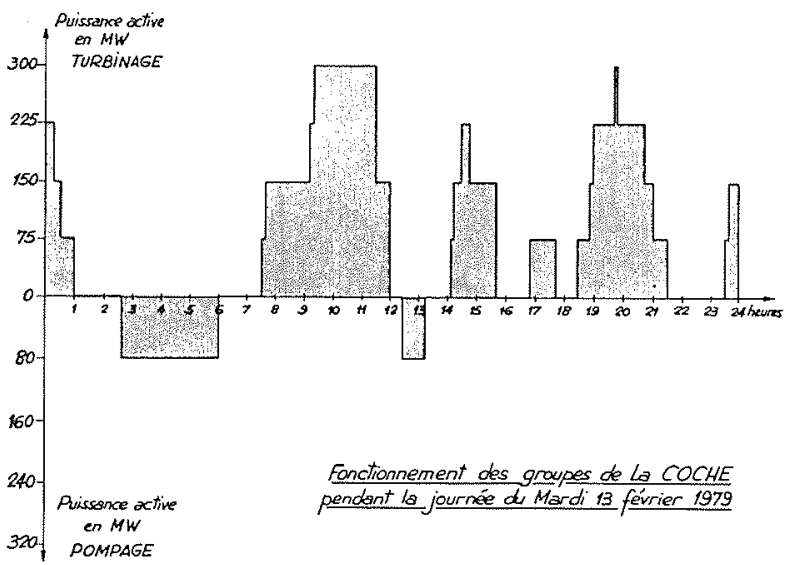

Figure 3 - Fonctionnement des groupes de la Coche pendant la journée du Mardi 13 février 1979
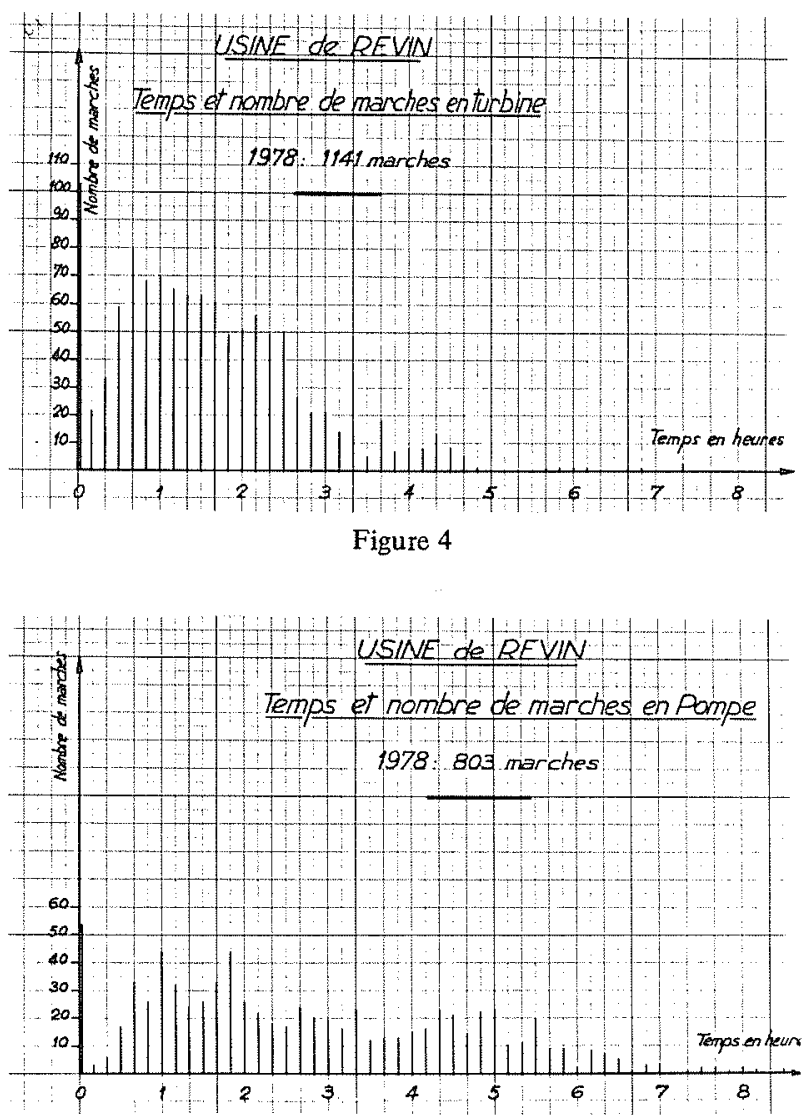

Figure 5

comportant pas de vannage, fonctionnent en turbine par tout ou rien et ne peuvent fonctionner en compensateur.

Ces fonctionnements de courte durée apparaissent également lorsqu'on trace ce que l'on pourrait appeler la "courbe des temps de marche classés". Sur les figures 4 et 5 relatives à la Centrale de Revin, on voit que les durées de marche les plus fréquentes sont de l'ordre de 1 à $2 \mathrm{~h}$ aussi bien en pompe qu'en turbine, mais il y a eu un nombre non négligeable de fonctionnements de courte durée $(21$ marches en turbine de 10 minutes et même 103 marches de très courte durée inférieures à 10 minutes).

Ces modes de fonctionnement découlent des besoins actuels des Services Utilisateurs des Mouvements d'Energie qui, la plupart du temps, télécommandent à distance les groupes de ces centrales.

En effet, ces Services assignent à ces centrales les rôles suivants :

Secours immédiat suite à un déclenchement imprévu d'une Unité thermique de grosse puissance ou d'une défaillance de ligne d'interconnexion internationale par exemple. Ce secours doit être assuré dans les 5 minutes et met en jeu la fourniture de la puissance maximale possible de tous les groupes disponibles de la Centrale. Ceci conduit à concevoir des machines ayant un temps d'intervention réduit, en particulier pour les marches en turbine.

La figure 6 montre les temps réels de démarrage et de transition d'une marche à l'autre pour les groupes de Revin. On voit que le temps de passage à la marche en 


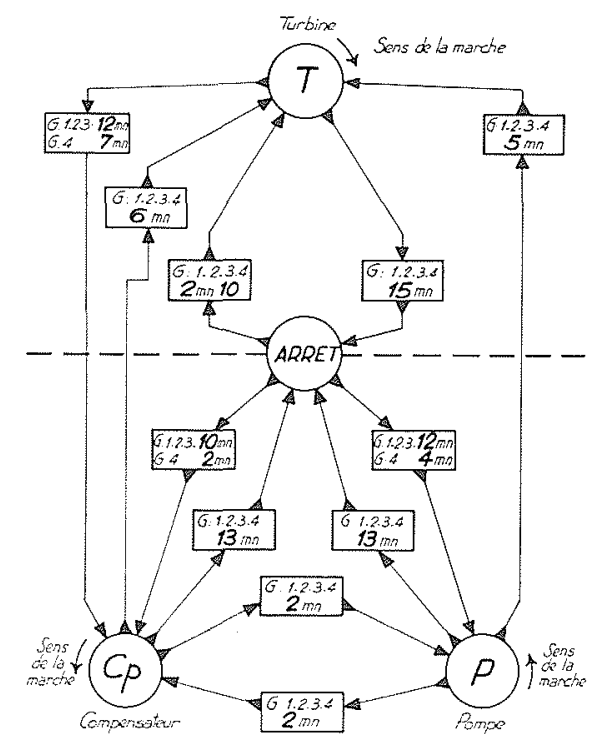

Figure 6 - Schéma de fonctionnement des groupes de Revin

turbine est de $2 \mathrm{mn} 10 \mathrm{~s}$ depuis l'arrêt, de $5 \mathrm{mn}$ depuis la marche en pompe et $6 \mathrm{mn}$ depuis la marche en compensateur.

Les transitions vers la marche en turbine, en partant de l'état pompe ou compensateur synchrone, sont un peu longues eu égard aux souhaits des exploitants. Un temps d'intervention en turbinage de l'ordre de $2 \mathrm{mn}$ à partir de tous les états possibles, et jusqu'à la pleine puissance, serait souhaitable. Les groupes ternaires répondent d'ailleurs à un tel critère.

Pour les groupes réversibles, l'utilisation du freinage électrique ou hydraulique qui consiste à feiner la pompe par réouverture du distributeur avant inversion de la rotation devrait permettre d'approcher cette valeur. A Revin, le freinage hydraulique est exploité, mais avec une grande prudence, de sorte que la transition de marche est encore longue.

La marche synchrone dans le sens turbine (non prévue à Revin) pourrait également améliorer le temps d'intervention si l'on utilise une technique de renoyage proche de celle utilisée sur les groupes Francis : éjection de la poche d'air vers l'aval. Cette solution n'est évidemment envisageable que dans les installations disposant d'une cheminée aval proche des machines et bien étudiée pour piéger le volume d'air sans à-coup de pression.

L'absence de cheminée aval rend problématique l'intérêt de la marche synchrone en turbinage pour accroitre la promptitude de prise de charge. En effet, le renoyage de la machine à distributeur fermé est rendu difficile par la forme des aubages qui écopent l'anneau d'eau et tendent à entretenir une émulsion. Le critère de fin de renoyage est alors difficile à saisir.

On ne saurait trop inciter les constructeurs à étudier ces problèmes, et à améliorer autant que possible la promptitude du passage des groupes réversibles en turbinage.

\section{Soutien du réseau et modulation de la production}

Ce rôle, qui consiste à un ajustement "en temps réel" de la production à la consommation, est surtout impor- tant en hiver. Il consiste à absorber ou produire de l'énergie avec les groupes de pompage de façon à "coller" le plus possible à la courbe de consommation : - en prévoyant les marches pompes ou turbines à l'avance, et lorsqu'ils fonctionnent en turbine : - en les soumettant au réglage primaire par l'intermédiaire de leur régulateur ;

- en les mettant sous la dépendance du téléréglage effectuant le réglage secondaire.

Outre les changements de marche, démarrages ou arrêts, ce rôle de modulation de la puissance produite provoque comme d'ailleurs dans les Centrales de lac des mouvements incessants des organes de réglage, vannages en particulier.

Fonctionnement "économique" à savoir Transfert d'Energie d'heures creuses en heures pleines de façon à réaliser une opération rentable en jouant sur les coûts différentiels.

Sans minimiser ce rôle qui semblait prépondérant il y a quelques années, et en particulier lors des études de conception de ces Centrales, nous avons l'impression qu'il est actuellement secondaire compte tenu de la structure du parc de production, ce qui d'ailleurs pourra être modifié par l'accroissement de la Production Nucléaire.

En conclusion nous voyons que les deux premières utilisations dont j'ai parlé : secours immédiat et soutien du réseau par modulation de la puissance provoquent une très nette évolution dans le fonctionnement des pompes turbines vers les marches fréquentes et de courte durée, et ceci particulièrement en hiver.

\section{Conséquence de ces modes de fonctionnement pour le matériel électromécanique}

Je distinguerai dans mon analyse les problèmes touchant le gros matériel hydraulique et électrique, et ceux concernant ce que nous pourrions appeler le matériel périphérique des groupes, en particulier les capteurs et les relayages.

\section{Gros matériel électromécanique}

La pompe-turbine proprement dite ne paraît pas souffrir outre mesure de ces sollicitations. On peut toutefois signaler :

- des usures par cavitation sur certaines pales à l'entrée en pompe, résultant en première analyse de défauts de forme localisés,

- des problèmes de joints d'étanchéité, en particulier au niveau des tourillons de directrices.

Les écoulements dans la roue pendant les marches transitoires fréquentes n'ont, à notre connaissance, aucune conséquence, ce qui paraît normal compte tenu de leur faible durée.

Si la phase de démarrage en pompe a posé des problèmes à Revin, et d'ailleurs dans d'autres centrales étrangères, les modifications effectuées ont, semble-t-il, résolu le problème.

Le pivot des pompes-turbines s'est avéré, dans certains cas, difficile à mettre au point sans doute du fait 
des 2 sens de marche et des charges spécifiques variables, mais ne pose plus de problème après cette mise au point.

Le joint plan vraisemblablement pour les mêmes raisons et du fait de la contre-pression élevée et variable, reste un organe délicat.

Les sollicitations dues aux vibrations sur les organes de la pompe-turbine tels que l'aspirateur, en particulier lors des séquences de barbotage avant l'ouverture du distributeur en pompe, doivent être prises en compte lors des études de conception, surtout dans le cas où le cône d'aspiration n'est pas noyé dans le béton pour permettre le démontage de la roue par-dessous, disposition qui présente de réels avantages en exploitation.

$L$ 'alternateur-moteur ne pose des problèmes que lorsqu'est dévolu à cet organe le rôle de lanceur du groupe en pompe par couplage direct sur le réseau après une phase asynchrone. Ce type de démarrage qui conduit à des appels de courant importants provoque des efforts électromagnétiques sur le bobinage stator et des échauffements importants des cages d'amortisseur rotorique.

Les principaux problèmes qui en découlent concernent :

- le calage et la fixation des développantes et le calage des barres dans les encoches qui doivent être parfaits, de manière à éviter les vibrations génératrices d'usure des isolants ;

- la tenue dans le temps des cages d'amortisseurs du rotor et des cercles de fermeture de ces amortisseurs. Ces problèmes difficiles á régler nous incitent à écarter les démarrages en pompe en asynchrone, pourtant économiques au point de vue investissement, au profit des démarrages par moteur Poney ou par convertisseur de fréquence ou en dos-à-dos.

Ces points délicats concernant les alternateursmoteurs et qui se ramènent toujours à des problèmes de tenue mécanique dans le temps des matériaux constitutifs de l'alternateur, sont aggravés par les cycles de température dûs aux fonctionnements de durée variable à charge variable de la machine. Ces fonctionnements sont générateurs de contraintes et de jeux. C'est ainsi que la conception des pôles rotors doit tenir compte des marches à excitation et température très variables.

Le freinage mécanique semble requérir, du fait de la reversibilité, une pression spécifique notablement plus basse, et, partant, un surdimensionnement, sous peine d'arrachement de la piste. Le rappel par ressorts des pistons de vérins semble également devoir être remplacé par un rappel hydraulique beaucoup plus puissant.

Les transformateurs associés aux groupes de pompage-turbinage ont posé des problèmes dans certaines centrales. Les cycles de température et les efforts électromagnétiques dus aux démarrages asynchrones lorsqu'ils existent sont certainement des éléments défavorables à leur tenue dans le temps. Nous pensons que les démarrages asynchrones ont également, sur ce type de matériel, un effet néfaste par les conséquences de la modulation à basse fréquence variable du courant d'appel pouvant engendrer des résonnances sur des fréquences propres des parties constitutives du transformateur.

Les organes de coupures hydrauliques : vannes sphériques la plupart du temps, sont très sollicités dans une centrale de Pompage-Turbinage. Nous avons eu de nombreux problèmes touchant ces organes, la plupart $\mathrm{du}$ temps concemant les joints mobiles, soit des problèmes de grippage, soit des problèmes de vibrations, en particulier lorsque ces anneaux mobiles ont un rôle de réglage comme à $\mathrm{La}$ Coche où les robinets permettent de régler le débit à vide lors du couplage.

Les Constructeurs doivent tenir compte de la très forte utilisation de ces organes qui remplissent un rôle de coupure de sécurité et qui doivent, de ce fait, avoir un fonctionnement irréprochable.

Les organes de coupure électriques sont encore plus sollicités que dans nos centrales de pointe et les disjoncteurs et sectionneurs doivent être d'un type dont l'endurance ne fait plus de doute.

L'essai de 10000 manœuvres que nous faisons subir aux prototypes des modèles choisis pour nos centrales classiques paraît insuffisant pour du matériel devant équiper des centrales de transfert. En effet, ces 10000 manceuvres ne représentent dans l'exploitation actuelle que 5 ans environ.

Remarquons que les problèmes touchant ce type de matériel sont toujours d'ordre mécanique, les intensités coupées en fonctionnement normal étant faibles.

\section{Matériel périphérique}

Nous abordons là un problème important car la défaillance de ce type de matériel, s'il n'a pas la plupart du temps de caractère de gravité, est la cause la plus fréquente des indisponibilités de ce type de machines.

Et ceci est très irritant pour l'Exploitant qui voit son groupe immobilisé ou plus souvent refuser simplement de démarrer à cause du mauvais fonctionnement d'un composant de prix réduit et de construction pas toujours adaptée au service attendu. Nous devons dire que les Constructeurs sont parfaitement conscients du problème, et qu'ils ont même sollicité notre aide pour effectuer un difficile choix parmi ces matériels que la plupart du temps ils ne fabriquent pas. Ajoutons que des essais systématiques qui ont été effectués sur certains composants : relais, fins de course, manostats, etc..., ne sont pas toujours significatifs dans la mesure où il est difficile de prévoir les contraintes réelles de fonctionnement en exploitation.

En outre, la probabilité de défaillance de ces composants est aggravée par leurs sollicitations fréquentes et leur grand nombre.

C'est ainsi qu'il y a à Revin :

139 manostats

94 thermostats

355 fins de course

138 détecteurs de niveaux

43 détecteurs de circulation soit 769 capteurs

3821 relais dont 293 temporisés.

Ces chiffres prouvent que ce type de centrale comporte environ 3 fois plus de composants qu'une centrale classique.

Un choix difficile doit être fait au moment de la conception du schéma. Doit-on compter sur des redondances pour lever des doutes en cas de mauvais fonctionnement d'un composant? Ceci conduit à augmenter leur nombre et à adopter une logique d'arbitrage, ce qui ne va pas dans le sens de la simplicité du schéma. Il faut bien 
avouer que ce type de Centrale justifie actuellement, par la complexité des schémas, l'utilisation de calculateur d'automatisme. C'est l'orientation qui est actuellement prise aussi bien en France qu'à l'Etranger.

\section{Principaux problèmes posés par ce "matériel péri- phérique"}

- Les vannes motorisées, nombreuses (14 par groupe à Revin) sur les circuits by-pass, réfrigération, dénoyage, vidange, adduction, etc. . ., doivent être d'une construction très robuste, surabondantes par rapport aux pressions de service et utilisant très largement l'acier inoxydable.

Notre préférence pour les pressions et sections courantes va aux petits sphériques de conception plus satisfaisante que les vannes à opercules.

Il faut remarquer que la fiabilité d'un certain nombre de cette vannes motorisées touche directement la sécurité de l'usine. C'est le cas des électrovannes de renoyage, de dégazage, qui peuvent provoquer, en cas de non fermeture, une inondation de l'usine à partir de la contrepression aval.

- les capteurs restent le point délicat de ce type de centrale et un gros effort doit être fait pour en améliorer la fiabilité. En particulier :

- les manostats, principalement ceux utilisés sur les circuits d'eau

- les fins de course dont les conditions de montage doivent être examinées avec soin

- les détecteurs de niveaux, en particulier ceux permettant le contrôle du niveau dans l'aspirateur pendant la phase de dénoyage lors des démarrages en pompe et des marches en compensateurs synchrones.

Ces derniers capteurs donnent des mécomptes à peu près dans toutes les centrales de pompage comportant un dénoyage, aussi bien à l'Etranger qu'en France.

La détection des niveaux s'effectuait à l'origine par un flotteur magnétique placé dans un circuit en dériva- tion de l'aspirateur. Ces capteurs trop rudimentaires compte tenu des vibrations et des fluctuations rapides de niveau qu'il est difficile d'amortir, pourraient etre avantageusement remplacés par des capteurs sans organe en mouvement, type ultra-sonique par exemple, mais leur installation doit être prévue dès l'origine et l'électronique associée doit être d'un fonctionnement irréprochable.

Nous effectuons actuellement à la Centrale de Revin une série d'essais avec divers appareils détecteurs de niveau pour trouver une solution à ce difficile problème.

Enfin, signalons l'importance des télétransmissions dans une telle centrale télécommandée ; en effet, toute panne remettant en cause l'exploitation à distance à partir du dispatching paralyse la centrale pour son rôle de secours immédiat.

\section{Conclusion}

Comme on peut le constater, les causes de défaillance dans une centrale de Pompage-Turbinage sont nombreuses. Certaines graves, mais heureusement peu fréquentes, touchent le gros matériel qui, du fait des très nombreux cycles d'exploitation, est soumis à des régimes transitoires sévères, générateurs de contraintes mécaniques pour le matériel hydraulique, auxquelles s'ajoutent des contraintes thermiques pour le matériel électrique.

D'autres défaillances moins graves mais malheureusement plus fréquentes touchent le matériel annexe mécanique et électique, capteurs en particulier. C'est surtout sur ce dernier point que l'attention des Constructeurs et des Maîtres d'Oeuvre devra porter dans les installations futures, pour que ce type de centrales puisse avoir une disponibilité qui permette d'utiliser leurs qualités de rapidité d'intervention et de modulation, qualités qui apparaissent aujourd'hui très importantes pour obtenir une bonne exploitation du réseau. 


\section{Discussion}

Président : M. J. CHEVALIER

Au cours de son exposé, M. LACOSTE dit quelques mots sur la panne du 19 décembre 1978 et sur la centrale de Revin.

M. le Président. - A ce sujet, une simulation est à l'étude par les Services d'Etudes de Réseaux de la Direction des Etudes et Recherches d'EDF qui inclut l'influence du déclenchement de Revin ou de son non déclenchement pour voir quelle influence ceci peut avoir sur le comportement du réseau. Je ne peux pas en dire davan tage actuellement.

Je remercie M. LACOSTE d'avoir fait toucher du doigt les aspects tout à fait particuliers de l'exploitation de ces centrales de pompage. Comme vous l'avez dit, cela peut faire sourire certains de nos collègues de la Production Thermique quand vous énumérez le nombre de vannes motorisées de Revin. Quand vous évoquez la vanne qui reste ouverte et qui menace l'inondation de la centrale, cela doit rappeler d'autres incidents récents...

Est-ce que, sur ce sujet de l'exploitation des centrales de pompage, il y a des questions que vous souhaitez poser à M. LACOSTE ?

M. BERGERON. - Je voudrais in tervenir non sur une question d'exploitation, mais au sujet de l'existence d'érosion de cavitation sur la face cachée des aubes de pompe.

En premier lieu M. LACOSTE a fait la remarque que les phénomènes constatés n'avaient affecté que certaines aubes, mais non toutes.

Or des érosions dues à des écoulements hydrauliques apparaissent sur toutes les aubes et dans la même région. Dans le cas particulier, il faudrait donc attribuer les phénomènes à une non identité de l'exécution des aubes les unes par rapport aux autres, par exemple : forme de l'arête d'entrée, congé, etc...

Comme deuxième remarque je dirais que personnellement je n'ai jamais vu d'érosions sur la face cachée des aubes de pompes depuis 50 ans que j'observe ce genre de machine.

Pour justifier cette constatation, il y a une tentative d'explication.

Même en surdébit, à la cavitation éventuelle apparaissant sur la face cachée succède rapidement une surpression supérieure à la dépression existante sur la paroi vue qui lui fait face. Comme les bulles glissent vers les zones de dépression, celles qui se sont dégagées dans la zone de cavitation ont une tendance à s'échapper dans la masse de l'écoulement sans imploser sur la paroi cachée dont elles s'éloignent.

De toute manière, elles ne pourraient éroder cette paroi qu'à une đistance notable de l'arête d'entrée, ce qui n'est pas le cas à Revin.

M. LACOSTE. - Les traces de cavitation dont j'ai parlé se situent bien à l'entrée en pompe sur les faces cachées des aubes. Il semble bien qu'elles proviennent d'un défaut de forme d'ailleurs visible à l'œil sur certaines aubes.

Il faut signaler que ces cavitations qui se produisent à fort débit, augmentent très vite avec la vitesse, donc avec le débit. Elles sont donc aggravées lorsque la machine présente un surdébit par rapport au débit nominal prévu, comme cela s'est passé sur la plupart des pompes-turbines installées à ce jour.

M. le Président remercie M. LACOSTE.

La séance est levée à $17 \mathrm{~h} .45$.
Génissiat (1950) - - $296 \mathrm{p}$ 413 p. . . . . . . . . . . . . . . . Bort (1953). -367 p. . . . . . . . . . . . Donzère-Mondragon (1955) $-486 \mathrm{p} . \ldots \ldots \ldots$ Le Marais Vernier (1957),-60 p. . . . . . . . . . . . . Tignes (1958). - 440 p. . . . . . . . . . . . Influence des rejets thermiques sur le milieu vivant en mer et en estuaire. (Journées de la Thermo-Ecologie-Centre Océanologique de Bretagne - Novembre 1976). -622 p. . . .

SOCIETE HYDROTECHNIQUE DE FRANCE / ASSOCIATION INTERNATIONALE DE RECHERCHES HYDRAULIQUES

Recherches sur les turbines hydrauliques (Symposium de Nice, 16-20 septembre 1960) - 431 p. (repr. Xérox) .....
$150 \mathrm{~F}$

$200 \mathrm{~F}$

$175 \mathrm{~F}$

$250 \mathrm{~F}$

$60 \mathrm{~F}$

$210 \mathrm{~F}$

$200 \mathrm{~F}$

$460 \mathrm{~F}$
SOCIETE HYDROTECHNIQUE DE FRANCE: JOURNEES DE L'HYDRAULIQUE

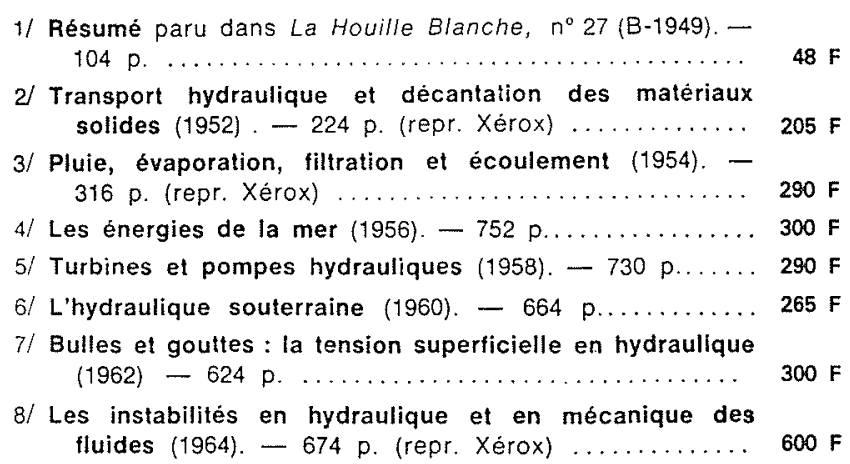

